\title{
NON-SINGULAR ASSEMBLY MODE CHANGING TRAJECTORIES OF A 6-DOF PARALLEL ROBOT
}

\author{
Stéphane Caro, Philippe Wenger, Damien Chablat \\ Institut de Recherche en Communications et Cybernétique de Nantes (IRCCyN) \\ UMR CNRS n ${ }^{\circ} 6597$ \\ 1, rue de la Noë, 44321 Nantes Cedex 03, France \\ Email addresses: \{stephane.caro, philippe.wenger, damien.chablat\}@irccyn.ec-nantes.fr
}

\begin{abstract}
This paper deals with the non-singular assembly mode changing of a six degrees of freedom parallel manipulator. The manipulator is composed of three identical limbs and one moving platform. Each limb is composed of three prismatic joints of directions orthogonal to each other and one spherical joint. The first two prismatic joints of each limb are actuated. The planes normal to the directions of the first two prismatic joints of each limb are orthogonal to each other. It appears that the parallel singularities of the manipulator depend only on the orientation of its moving platform. Moreover, the manipulator turns to have two aspects, namely, two maximal singularity free domains without any singular configuration, in its orientation workspace. As the manipulator can get up to eight solutions to its direct kinematic model, several assembly modes can be connected by nonsingular trajectories. It is noteworthy that the images of those trajectories in the joint space of the manipulator encircle one or several cusp point(s). This property can be depicted in a three dimensional space because the singularities depend only on the orientation of the moving-platform and the mapping between the orientation parameters of the manipulator and three joint variables can be obtained with a simple change of variables. Finally, to the best of the authors' knowledge, this is the first spatial parallel manipulator for which non-singular assembly mode changing trajectories have been found and shown.
\end{abstract}

\section{INTRODUCTION}

Because at a singularity a parallel manipulator loses its stiffness, it is of primary importance to be able to characterize these special configurations. This is, however, a very challenging task for a general parallel manipulator [1-3].

The direct and inverse kinematic problem of parallel robots have been study in many papers to define first the maximal numbers of solution(s) for each problem and secondly to characterize the joint space and workspace. The moving platform can admit several positions and orientations (or configurations) in the workspace for one given set of input joint values. Conversely, the robot can admit several input joint values for a given moving platform configuration.

The notion of assembly modes was defined to represent the different solutions to the direct kinematic problem while the notion of working mode was introduced to separate the solutions to the inverse kinematic problem [4].

To cope with the existence of multiple inverse kinematic solutions in serial mechanisms, the notion of aspects was introduced [5]. The aspects were defined as the maximal singularityfree domains in the joint space. The same notion was extended for parallel mechanism with several inverse and direct kinematic solutions $[4,6]$.

For path planning, we need to define a one-to-one mapping between the joint space and the workspace, which makes it possible to associate one single solution to the inverse and direct kinematic problem. One way to solve this problem is to introduce the definition of the uniqueness domains. Like for serial mechanisms, the aspects do not define the uniqueness domains of the inverse and direct kinematic problem because some parallel robots are able to change assembly-mode without passing through a singularity, thus meaning that there is more than one direct kinematic solution in one aspect [7]. This feature was first analyzed for the 3-RPR parallel robot and more recently for other ones such as the RPR-2PRR [8]. 
It was first pointed out that to move from one assembly mode to another, the manipulator should cross a singularity [5,9]. Later, Innocenti and Parenti-Castelli [10] showed, using numerical experiments, that this statement is not true in general. In fact, this statement is only true under some special geometric conditions, such as similar base and moving platforms $[11,12]$ for planar parallel manipulators. More recently, Macho et al. [13] proposed a method to plan non-singular assembly-mode changing trajectories. McAree [11] pointed out that for a 3-RPR parallel manipulator, if a point with triple direct kinematic solutions exists in the joint space, then the non-singular change of assembly mode is possible. This result holds under some assumptions on the topology of the singularities [14].

The change of assembly-mode was first analyzed in the joint space, but did not make it possible to explain the nonsingular assembly-mode phenomenon. To solve this problem, a configuration-space was defined by the input joint value plus one coordinate of the platform configuration [15]. This approach makes it possible to show that a cusp point may be encircled during a non-singular assembly-mode motion. A second problem is to find trajectories that induce an assembly mode changing. This problem can be solved by defining the uniqueness domains as defined for serial robots in [16] and for parallel robots in [17]. For other mechanisms than 3-RPR manipulator, it is also interesting to note that encircling a cusp point is not the only way to execute a non-singular change of assembly mode [18].

This paper deals with the non-singular assembly mode changing of a six degrees of freedom parallel manipulator presented in [19]. The manipulator is composed of three identical limbs and one moving platform. Each limb is composed of three prismatic joints of directions orthogonal to each other and one spherical joint in series. The first two prismatic joints of each limb are actuated. The planes normal to the directions of the first two prismatic joints of each limb are orthogonal to each other.

The paper is organized as follows. Section 2 describes the manipulator under study and its parameterization. Section 3 deals with its singularity analysis. Section 4 presents the cusp points of the manipulator in its joint space. Finally, two nonsingular assembly mode changing trajectories are highlighted in Sec. 5.

\section{MANIPULATOR DESCRIPTION}

Figure 1 illustrates the parallel manipulator under study, which is a simplified kinematic version of the manipulator proposed in [20]. It is composed of an equilateral moving platform connected to the base with three identical legs. Each leg is composed of three orthogonal prismatic joints and one spherical joint, the first two prismatic joints being actuated. $P$ stands for a prismatic joint whereas $S$ stands for a spherical joint. An underline letter denotes an actuated joint. As a consequence, the manipulator is named the 3-PPPS-manipulator and provides six-degree-

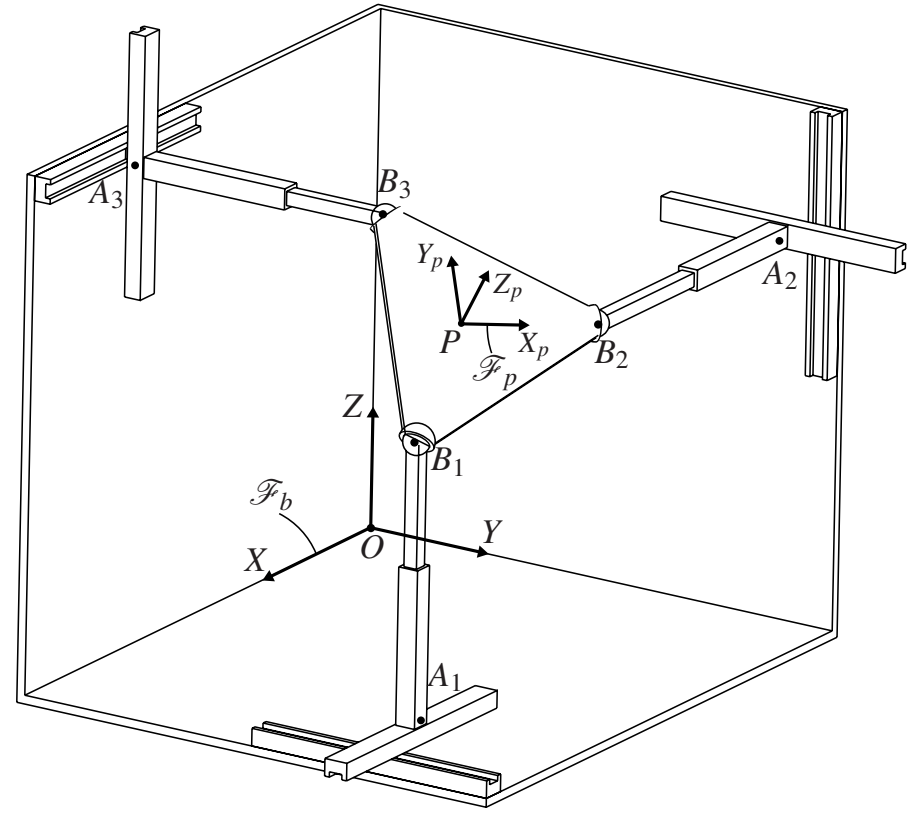

FIGURE 1. The 3-PPPS-manipulator

of-freedom motions, i.e., three translations and three rotations.

\subsection{Parameterization}

Let $B_{1}, B_{2}$ and $B_{3}$ be the corners of the moving platform (MP) of side length $r$. Let $\mathscr{F}_{p}\left(P, X_{p}, Y_{p}, Z_{p}\right)$ be the frame attached to the moving platform, its origin $P$ being the centroid of the MP. $Y_{p}$ is parallel to line $\left(B_{1} B_{3}\right)$ and $Z_{p}$ is normal to the MP. Accordingly,

$$
\mathbf{b}_{1 p}=\left[\begin{array}{c}
-r \sqrt{3} / 6 \\
-r / 2 \\
0
\end{array}\right], \mathbf{b}_{2 p}=\left[\begin{array}{c}
2 r \sqrt{3} / 6 \\
0 \\
0
\end{array}\right], \mathbf{b}_{3 p}=\left[\begin{array}{c}
-r \sqrt{3} / 6 \\
r / 2 \\
0
\end{array}\right]
$$

are the Cartesian coordinate vectors of points $B_{1}, B_{2}$ and $B_{3}$ expressed in $\mathscr{F}_{p}$. Likewise, let $\mathscr{F}_{b}(O, X, Y, Z)$ be the frame attached to the base and

$$
\mathbf{a}_{1 b}=\left[\begin{array}{c}
x_{1} \\
y_{1} \\
0
\end{array}\right], \quad \mathbf{a}_{2 b}=\left[\begin{array}{c}
0 \\
y_{2} \\
z_{2}
\end{array}\right], \quad \mathbf{a}_{3 b}=\left[\begin{array}{c}
x_{3} \\
0 \\
z_{3}
\end{array}\right]
$$

be the Cartesian coordinate vectors of points $A_{1}, A_{2}$ and $A_{3}$ expressed in $\mathscr{F}_{b}$.

\subsection{Geometric Model}

Let $\mathbf{p}=\left[\begin{array}{lll}p_{x} & p_{y} & p_{z}\end{array}\right]^{T}$ be the Cartesian coordinate vector of point $P$, the centroid of the MP, expressed in $\mathscr{F}_{b}$ and let $r$ be 
equal to 1 . The orientation space of the moving platform is fully represented with the variables $(\phi, \theta, \sigma)$, namely, the azimuth, tilt and torsion angles defined in [21]. The rotation matrix ${ }^{b} \mathbf{Q}_{p}$ from $\mathscr{F}_{b}$ to $\mathscr{F}_{p}$ is expressed as follows:

$$
{ }^{b} \mathbf{Q}_{p}=\left[\begin{array}{ccc}
C_{\phi} C_{\psi}-S_{\phi} C_{\theta} S_{\psi} & -C_{\phi} S_{\psi}-S_{\phi} C_{\theta} C_{\psi} & S_{\phi} S_{\theta} \\
S_{\phi} C_{\psi}+C_{\phi} C_{\theta} S_{\psi} & -S_{\phi} S_{\psi}+C_{\phi} C_{\theta} C_{\psi} & -C_{\phi} S_{\theta} \\
S_{\theta} S_{\psi} & S_{\theta} C_{\psi} & C_{\theta}
\end{array}\right]
$$

$C$ and $S$ denoting the cosine and sine functions, respectively. Note that $\phi \in[-\pi, \pi], \theta \in[0, \pi]$ and $\sigma \in[-\pi, \pi]$.

As a consequence, the following constraint equations characterize the geometric model of the 3-PPPS-manipulator and are obtained by considering the projection of the coordinates of points $B_{i}$ in the plane motion of the two actuated prismatic joints of the $i$ th leg, $i=1, \ldots, 3$ :

$$
\begin{array}{r}
p_{x}-x_{1}=0 \\
p_{y}-y_{1}=0 \\
3 p_{y}-3 y_{2}-2 \sqrt{3} C_{\theta} S_{\sigma}+2 \sqrt{3} C_{\phi}^{2} C_{\theta} S_{\sigma}+2 \sqrt{3} C_{\phi} C_{\sigma} S_{\phi} \\
-\sqrt{6} S_{\theta} C_{\sigma} C_{\phi}-\sqrt{6} S_{\theta} S_{\sigma} S_{\phi}-2 \sqrt{3} S_{\phi} C_{\theta} C_{\sigma} C_{\phi}-2 \sqrt{3} C_{\phi}^{2} S_{\sigma}=0 \\
3 p_{z}-3 z_{2}+\sqrt{3} C_{\theta} S_{\sigma}-\sqrt{3} C_{\phi}^{2} C_{\theta} S_{\sigma}-\sqrt{3} C_{\phi} C_{\sigma} S_{\phi} \\
-\sqrt{6} S_{\theta} C_{\sigma} C_{\phi}-\sqrt{6} S_{\theta} S_{\sigma} S_{\phi}+\sqrt{3} S_{\phi} C_{\theta} C_{\sigma} C_{\phi}-3 C_{\phi} S_{\sigma} S_{\phi} \\
+3 C_{\phi}^{2} C_{\theta} C_{\sigma}+3 C_{\sigma}-3 C_{\phi}^{2} C_{\sigma}+\sqrt{3} C_{\phi}^{2} S_{\sigma}+3 S_{\phi} C_{\theta} S_{\sigma} C_{\phi}=0 \\
6 p_{x}-6 x_{3}+\sqrt{3} C_{\theta} S_{\sigma}+2 \sqrt{3} C_{\phi}^{2} C_{\theta} S_{\sigma} \\
+2 \sqrt{3} C_{\phi} C_{\sigma} S_{\phi}-\sqrt{6} S_{\theta} C_{\sigma} C_{\phi} \\
-\sqrt{6} S_{\theta} S_{\sigma} S_{\phi}+3 \sqrt{2} S_{\theta} S_{\sigma} C_{\phi}-3 \sqrt{2} S_{\theta} C_{\sigma} S_{\phi}-2 \sqrt{3} S_{\phi} C_{\theta} C_{\sigma} C_{\phi} \\
+3 C_{\theta} C_{\sigma}-6 C_{\phi}^{2} C_{\theta} C_{\sigma}+6 C_{\phi} S_{\sigma} S_{\phi}-3 C_{\sigma}+6 C_{\phi}^{2} C_{\sigma}-2 \sqrt{3} C_{\phi}^{2} S_{\sigma} \\
-6 S_{\phi} C_{\theta} S_{\sigma} C_{\phi}+3 \sqrt{3} S_{\sigma}=0 \\
6 p_{z}-6 z_{3}+\sqrt{3} C_{\theta} S_{\sigma}-4 \sqrt{3} C_{\phi}^{2} C_{\theta} S_{\sigma}-4 \sqrt{3} C_{\phi} C_{\sigma} S_{\phi} \\
-\sqrt{6} S_{\theta} C_{\sigma} C_{\phi}-\sqrt{6} S_{\theta} S_{\sigma} S_{\phi}+3 \sqrt{2} S_{\theta} S_{\sigma} C_{\phi}-3 \sqrt{2} S_{\theta} C_{\sigma} S_{\phi} \\
+4 \sqrt{3} S_{\phi} C_{\theta} C_{\sigma} C_{\phi}+3 C_{\theta} C_{\sigma}+3 C_{\sigma}+4 \sqrt{3} C_{\phi}^{2} S_{\sigma}-3 \sqrt{3} S_{\sigma}=0
\end{array}
$$

\subsection{Decoupling Between the Translational and Rota- tional Motions of the Moving-platform}

It is noteworthy that the translational and rotational motions of the moving platform of the 3-PPPS-manipulator shown in Fig. 1 can be decoupled. In order to highlight this decoupling, the following change of variables can be used:

$$
\begin{aligned}
& x_{1}=X_{1}-X_{3} / 2 \\
& y_{1}=Y_{2}-Y_{1} / 2 \\
& y_{2}=Y_{2}+Y_{1} / 2 \\
& z_{2}=Z_{3}+Z_{2} / 2 \\
& x_{3}=X_{1}+X_{3} / 2 \\
& z_{3}=Z_{3}-Z_{2} / 2
\end{aligned}
$$

namely,

$$
\begin{aligned}
X_{1} & =\frac{x_{1}+x_{3}}{2} \\
X_{3} & =x_{3}-x_{1} \\
Y_{1} & =y_{2}-y_{1} \\
Y_{2} & =\frac{y_{1}+y_{2}}{2} \\
Z_{2} & =z_{2}-z_{3} \\
Z_{3} & =\frac{z_{2}+z_{3}}{2}
\end{aligned}
$$

It is apparent the translational motions of the MP depend only on variables $X_{1}, Y_{2}$ and $Z_{3}$, whereas its rotational motions depend only on variables $X_{3}, Y_{1}$ and $Z_{2}$.

\section{SINGULARITY ANALYSIS}

The singularity of the 3-PPPS-manipulator were analyzed in [19] using Grassmann-Cayley Algebra and Groebner Bases. It was shown that the parallel singularities of the manipulator at hand depend only on the orientation of its moving-platform. It means that the parallel singularities of the 3-PPPS-manipulator depend only on joint variables $X_{3}, Y_{1}$ and $Z_{2}$. Note that the orientation space was fully represented with a subset of the quaternions coordinates in [19]. Here, the orientation space is fully represented with angle $\phi$, called the azimuth, angle $\theta$ referred to as the tilt and $\sigma$ angle, called the torsion, as they lead to a simple expression for the parallel singularities of the manipulator in its orientation workspace, namely,

$$
-4 \sin \left(\frac{\theta}{2}\right)\left(\cos \left(\frac{\theta}{2}\right)\right)^{2}
$$

$$
\left(\sqrt{2} \sin (3 \phi-\sigma)\left(\sin \left(\frac{\theta}{2}\right)\right)^{3}-\cos \left(\frac{3 \theta}{2}\right) \cos (\sigma)\right)=0
$$

Figure 2 illustrates the singularity surfaces of the 3-PPPSmanipulator in its orientation workspace. The first factor of 


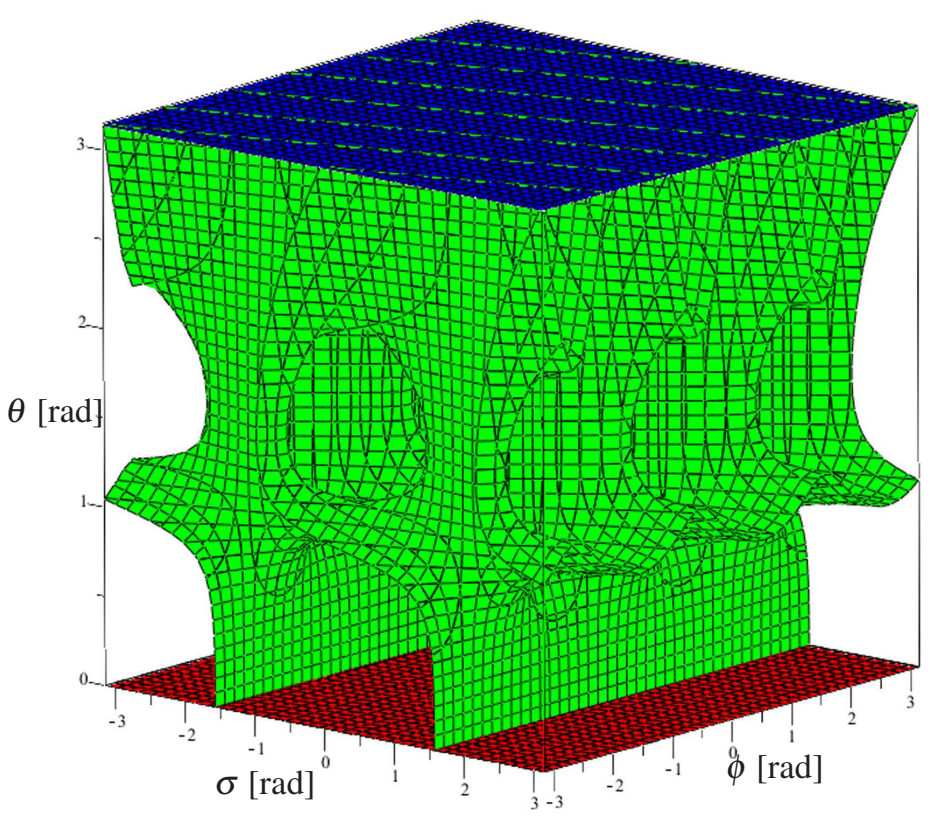

FIGURE 2. Singularity surfaces of the 3-PPPS-manipulator in its orientation workspace

Eq. (6), i.e., $\sin \left(\frac{\theta}{2}\right)$, is null on the red plane, $\theta=0$. The second factor of Eq. (6), i.e., $\cos \left(\frac{\theta}{2}\right)$, is null on the blue plane, $\theta=\pi$. The third factor of Eq. (6), i.e.,

$$
\sqrt{2} \sin (3 \phi-\sigma)\left(\sin \left(\frac{\theta}{2}\right)\right)^{3}-\cos \left(\frac{3 \theta}{2}\right) \cos (\sigma)
$$

is null on the green surface.

It is apparent that the green surface splits the orientation workspace of the manipulator into two connected parts. Those two parts amount to the two aspects of the 3-PPPS-manipulator.

The singularities of the 3-PPPS-manipulator can also be expressed in its joint space. An algebraic form of the singularities of the manipulator is given in Appendix A as a function of variables $X_{3}, Y_{1}$ and $Z_{2}$. The corresponding singularity surface is shown in Fig. 3.

Figure 4 depicts a slice of the singularity surface shown in Fig. 3 and obtained for $X_{3}=1 / 2$. Moreover, Fig. 4 shows five colored areas: $(i)$ the 3 -PPPS-manipulator does not have any solution to the direct kinematic model (DKM) in the white area; (ii) the 3-PPPS-manipulator has two solutions to the direct kinematic model for any joint coordinate vector $\left(Y_{1}, Z_{2}\right)$ selected in the red area; (iii) the 3-PPPS-manipulator has four solutions to the direct kinematic model for any joint coordinate vector $\left(Y_{1}, Z_{2}\right)$ selected in the yellow area; (iv) the 3-PPPS-

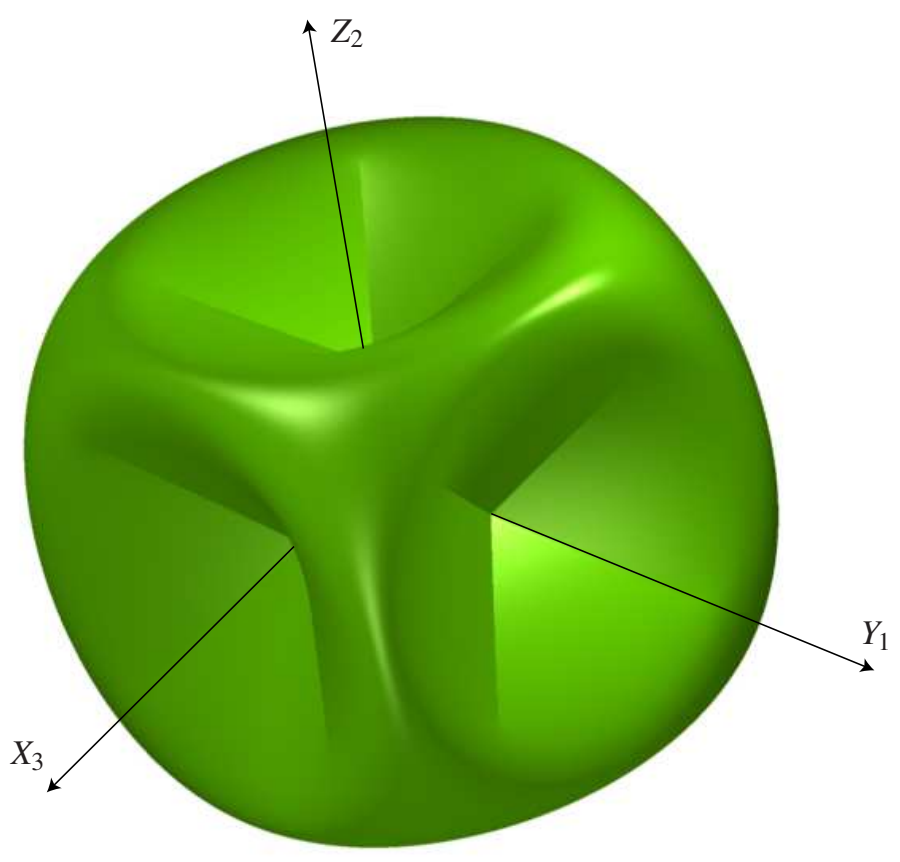

FIGURE 3. Singularity surface of the 3-PPPS-manipulator in the joint space defined by $X_{3}, Y_{1}$ and $Z_{2}$

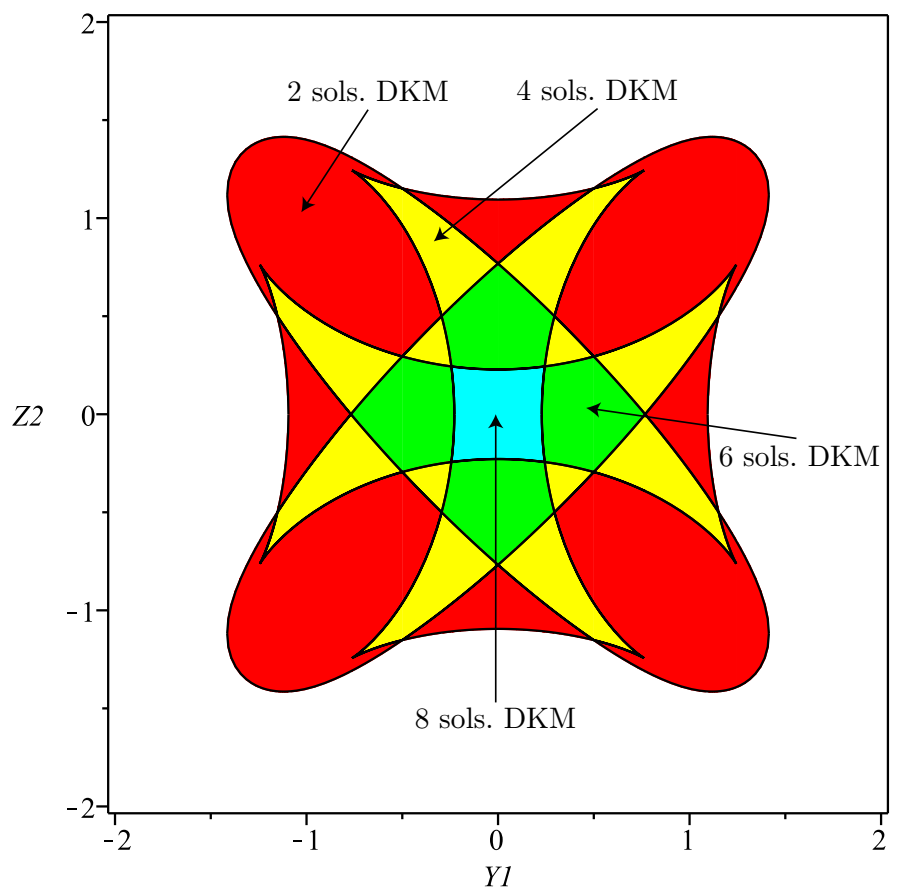

FIGURE 4. Slice of the joint space with the number of solutions to the direct kinematic model: 
manipulator has six solutions to the direct kinematic model for any joint coordinate vector $\left(Y_{1}, Z_{2}\right)$ selected in the green area; (v) the 3-PPPS-manipulator has eight solutions to the direct kinematic model for any joint coordinate vector $\left(Y_{1}, Z_{2}\right)$ selected in the cyan area.

The foregoing areas were obtained from the constraint equations given in Sec. 2.2 and with a method based on the notion of Discriminant Varieties and Cylindrical Algebraic Decomposition. This method resorts to Gröbner bases for the solutions of systems of equations and is described in [22,23]. Besides, the tools used to perform the computations are implemented in a Maple library called Siropa ${ }^{1}$

Let us notice that the 3-PPPS-manipulator can have eight solutions to its direct kinematic model while it has two aspects, namely, two maximal singularity free domains without any singular configuration, determined its orientation workspace. Therefore, some non-singular assembly mode changing trajectories exist for this manipulator.

\section{CUSPIDAL CONFIGURATIONS}

According to Moroz et al., cusp points play an important role in the kinematic behavior of parallel manipulators since they make possible a non-singular change of assembly mode [24]. This section aims to determine the cusp points of the 3-PPPSmanipulator in order to check whether the previous statement is true for this manipulator.

To find cuspidal configurations of a 3-R $\underline{P R}$ planar parallel manipulator, the idea of $[11,15]$ was to analyze the kernels of the matrices in the first and second order terms of the series expansion of the constraint equations of the manipulator. However it did not allow them to describe these configurations precisely. Using the notion of discriminant variety and a generalization of the Jacobian criterion, a complete certified description of the cuspidal configurations of the 3-R $\underline{P} R$ planar parallel manipulator at hand was introduced in [24]. This approach also allowed the authors to certify that all cuspidal configurations of the manipulator were determined.

This approach was implemented in the Siropa library under the function named ParallelCuspidal(). Consequenly, this function was used to obtain the cuspidal configurations of the 3-PPPS-manipulator from its constraint equations given in Sec. 2.2. The cuspidal configurations of the manipulator are expressed as a function of variables $X_{3}, Y_{1}$ and $Z_{2}$ in Appendix B. Finally, Fig. 5 represents the cusp points of the 3-PPPSmanipulator in its joint space. Figure 6 shows a slice of the singularity surface of the manipulator with 8 cusp points for $X_{3}=0.1$.

\footnotetext{
${ }^{1}$ http://www.irccyn.ec-nantes.fr/ chablat/SIROPA/files/siropa-mpl.html
}

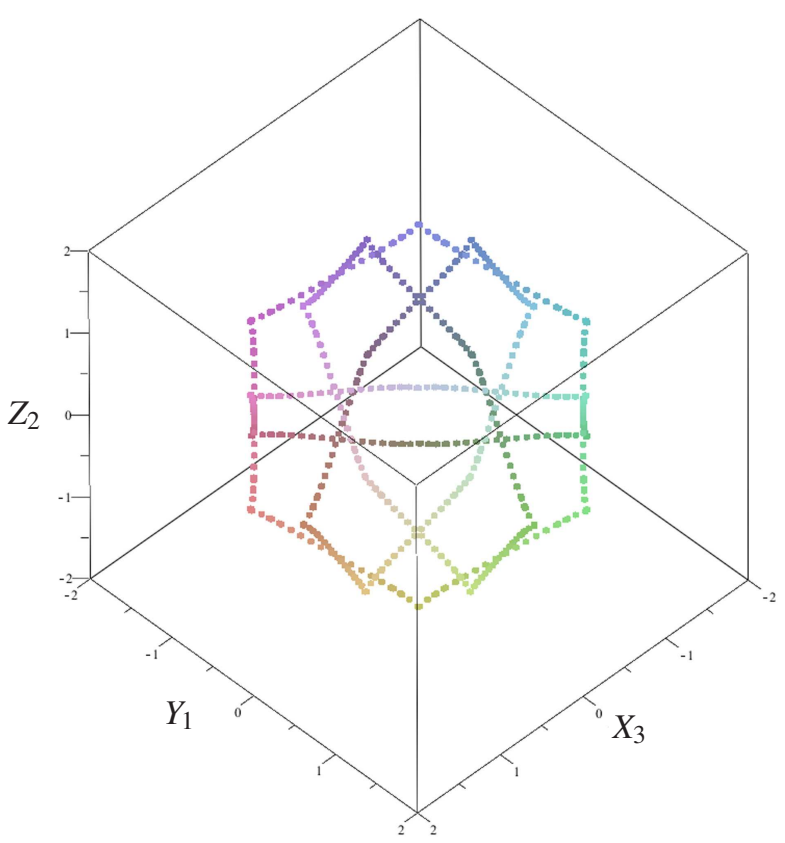

FIGURE 5. Cusp points of the 3-PPPS-manipulator

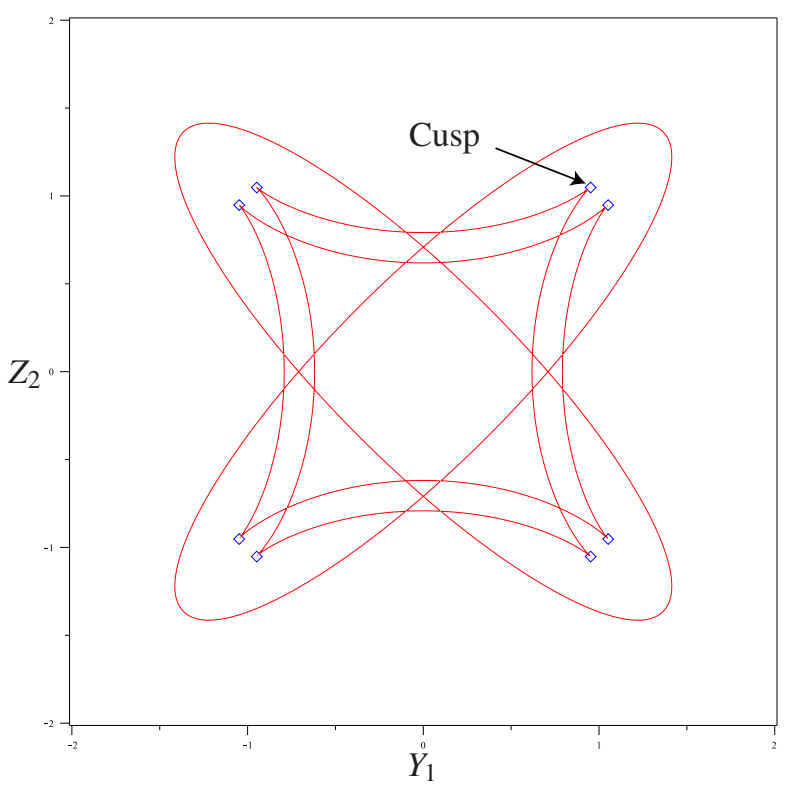

FIGURE 6. Slice of the singularity surface of the 3-PPPSmanipulator showing 8 cusp points for $X_{3}=0.1$

\section{NON-SINGULAR ASSEMBLY MODE CHANGING TRAJECTORIES}

As shown in Sec. 3, the orientation workspace of the 3-PPPS-manipulator can be split into two connected components, namely, two aspects. Therefore, some non-singular assembly mode changing paths can be found in both aspects. 
Figures 7 and 8 illustrate two paths $P_{1}-P_{2}-P_{3}-P_{4}$ and $P_{5}-P_{6}-$ $P_{7}-P_{8}$ plotted in each aspect.

Figures 9 and 10 represent the images of the foregoing paths in the joint space of the manipulator.

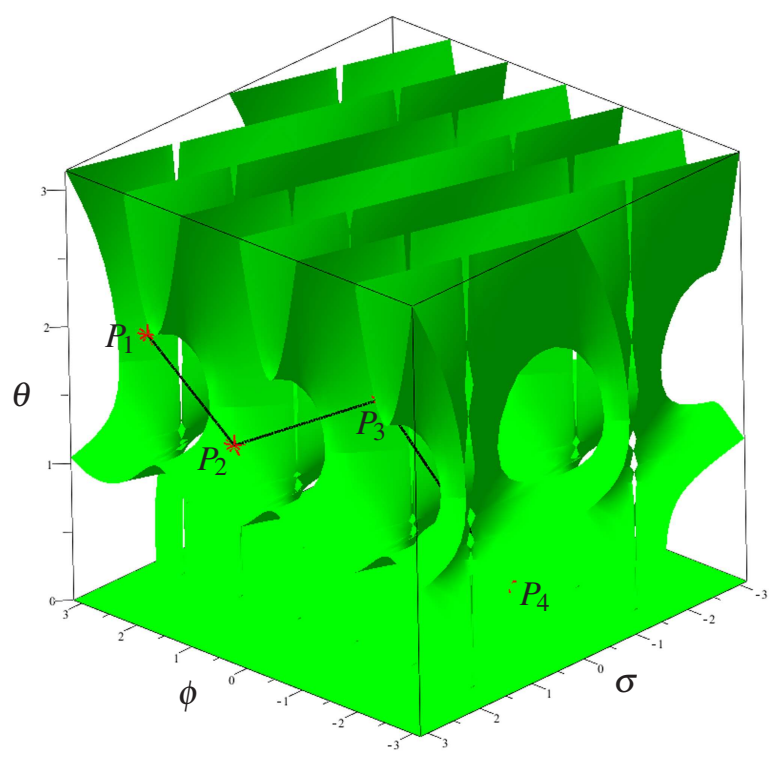

FIGURE 7. First non-singular assembly mode changing path $P_{1}-P_{2}-$ $P_{3}-P_{4}$ defined in the orientation workspace of the 3-PPPS-manipulator

Figures 11 and 12 depict the determinant $\operatorname{det}(\mathbf{J})$ of the kinematic Jacobian matrix $\mathbf{J}$ of the manipulator along the two paths. It is noteworthy that $\operatorname{det}(\mathbf{J})$ does not vanish along the two paths while the manipulator performs three assembly mode changing. It means that the 3-PPPS-manipulator does not meet any singularity when its moving platform follows the two paths $P_{1}-P_{2}-P_{3}-$ $P_{4}$ and $P_{5}-P_{6}-P_{7}-P_{8}$.

We can also notice in Figs. 9 and 10 that the two paths encircle three times the cusp points. Consequently, the statement of Moroz et al. [24] recalled in Sec. 4 seems to be also true for the 3-PPPS-manipulator but this remains to be formally shown.

The two non-singular assembly mode changing trajectories can be downloaded in ${ }^{2}$ and ${ }^{34}$.

\section{CONCLUSIONS}

This paper dealt with the non-singular assembly mode changing of the 3-PPPS-manipulator. The manipulator is composed of three identical limbs and one moving platform. Each

\footnotetext{
${ }^{2}$ http://www.irccyn.ec-nantes.fr/ caro/DETC2012/Traj1.avi ${ }^{3}$ http://www.irccyn.ec-nantes.fr/ caro/DETC2012/Traj2.avi

${ }^{4}$ Note that $\sim$ denotes the "tilde" symbol in the previous links.
}

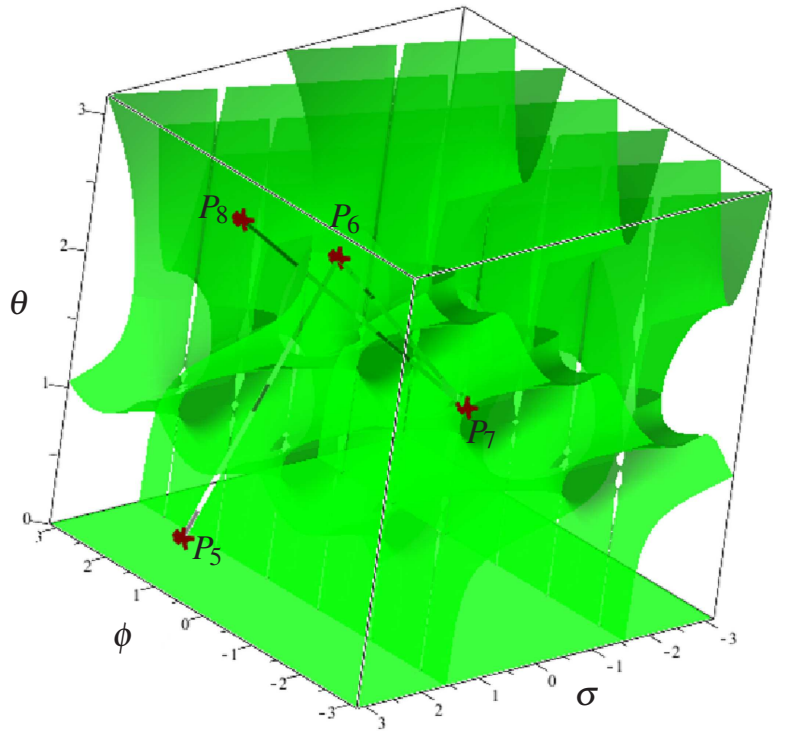

FIGURE 8. Second non-singular assembly mode changing path $P_{5}-P_{6}-P_{7}-P_{8}$ defined in the orientation workspace of the 3-PPPSmanipulator

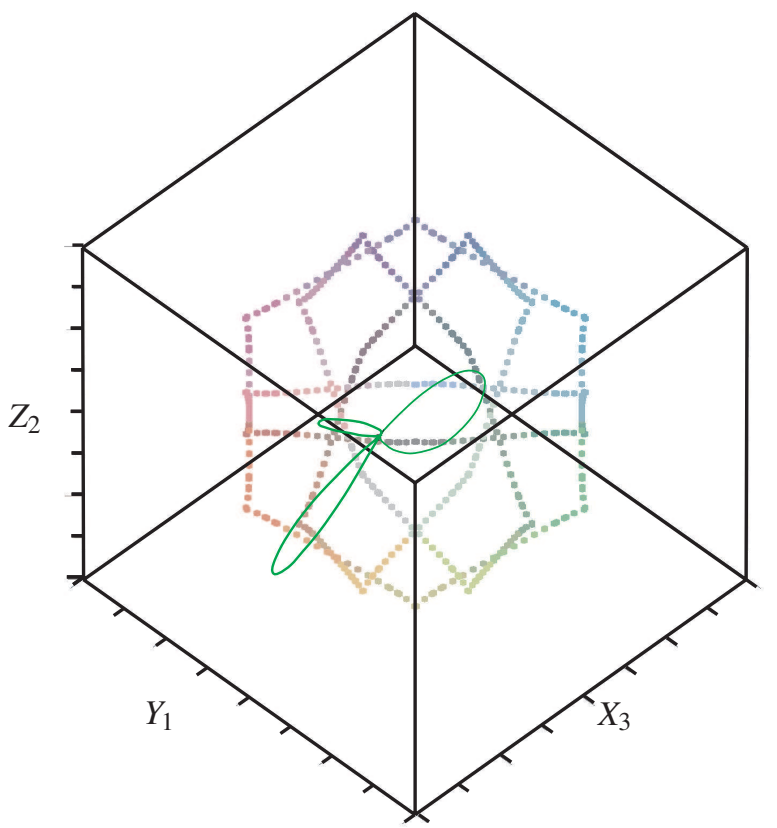

FIGURE 9. Image of the path $P_{1}-P_{2}-P_{3}-P_{4}$ in the joint space $\left(X_{3}, Y_{1}\right.$, $Z_{2}$ )

limb is composed of three prismatic joints of directions orthogonal to each other and one spherical joint. The first two prismatic joints of each limb are actuated. The planes normal to the directions of the first two prismatic joints of each limb are orthogonal 


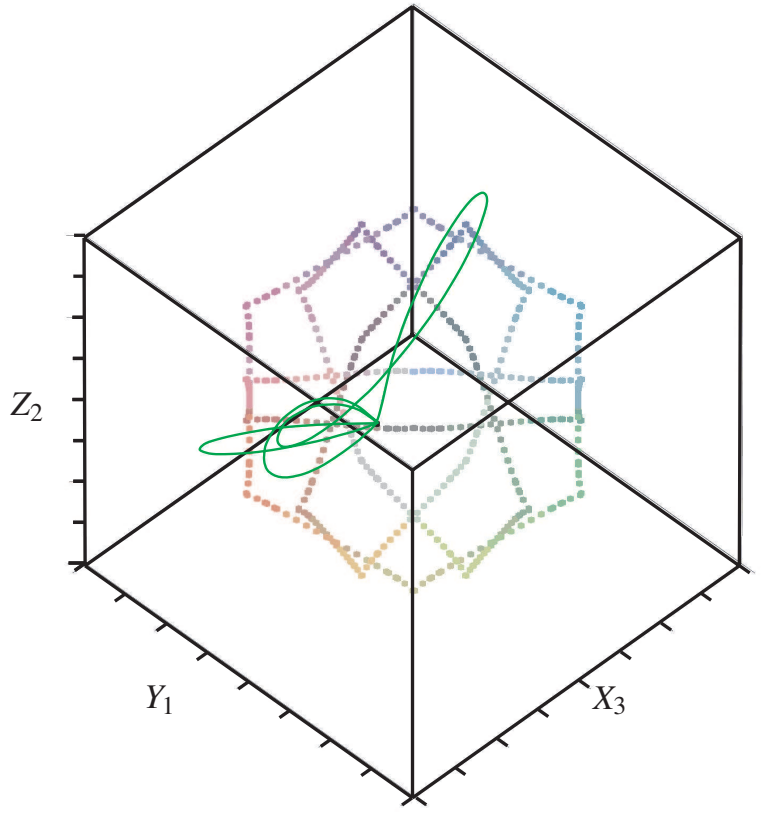

FIGURE 10. Image of the path $P_{5}-P_{6}-P_{7}-P_{8}$ in the joint space $\left(X_{3}\right.$, $\left.Y_{1}, Z_{2}\right)$

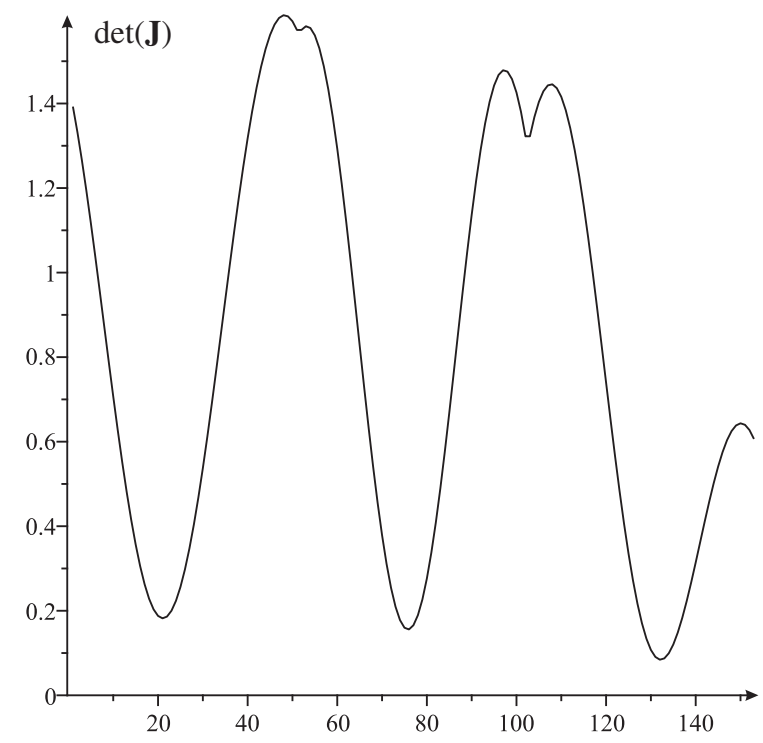

FIGURE 11. Determinant of the kinematic Jacobian matrix $\mathbf{J}$ of the 3-PPPS-manipulator along the path $P_{1}-P_{2}-P_{3}-P_{4}$

to each other.

It was shown that the parallel singularities of the manipulator depend only on the orientation of its moving platform. Moreover, the manipulator turned to have two aspects, namely, two maximal singularity free domains without any singular configu-

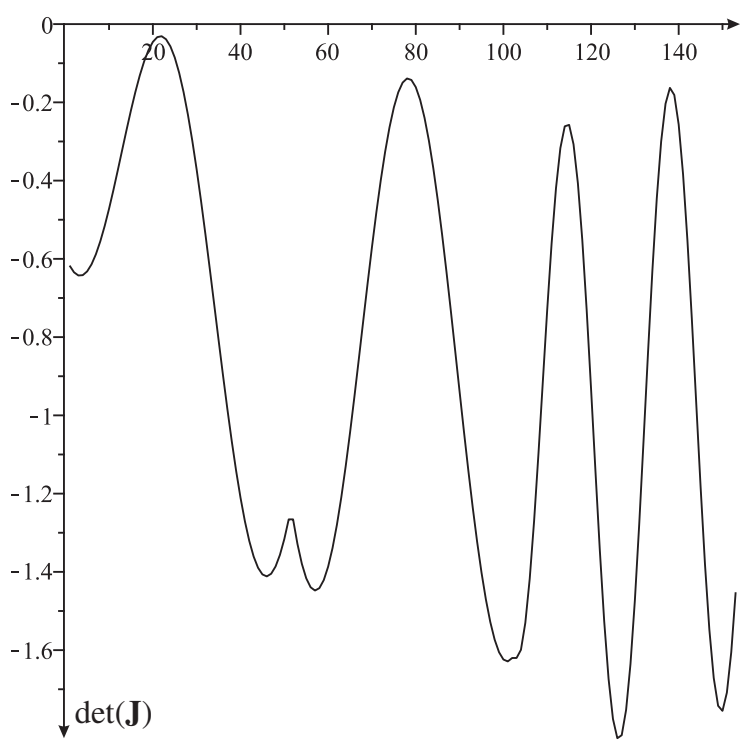

FIGURE 12. Determinant of the kinematic Jacobian matrix $\mathbf{J}$ of the 3-PPPS-manipulator along the path $P_{5}-P_{6}-P_{7}-P_{8}$

ration, in its orientation workspace.

As the manipulator can get up to eight solutions to its direct kinematic model, several assembly modes can be connected by non-singular trajectories. It is noteworthy that the images of the trajectories found in the joint space of the manipulator encircle three times the cusp points. As a consequence, cusp points seem to play an important role in the kinematic behavior of the 3-PPPS-manipulator as they apparently make possible a non-singular change of assembly mode. This property can be depicted in a three dimensional space because the singularities depend only on the orientation of the moving-platform and the mapping between the orientation parameters of the manipulator and three joint variables was obtained thanks to a simple change of variables.

Two illustrative non-singular assembly mode changing trajectories were highlighted in this paper. As the determinant of the kinematic Jacobian matrix of the manipulator does not vanish along those two trajectories, the 3-PPPS-manipulator under study does not meet any singularity when its moving-platform follows the corresponding trajectories, whereas the manipulator performs three assembly mode changing.

The two areas of the orientation workspace in which nonsingular assembly mode changing trajectories can be realized will be defined rigorously by using a cylindrical algebraic decomposition in a future work.

Finally, to the best of the authors' knowledge, this is the first spatial parallel manipulator for which non-singular assembly mode changing trajectories have been found and illustrated through some examples. 


\section{ACKNOWLEDGMENT}

The authors would like to acknowledge the financial support of the ANR, France (Project ANR-06-ROBO-0002-SIROPA) and the financial support of the Egide Programme FAST (FAST Project No 24154WA).

\section{REFERENCES}

[1] Bonev, I., Zlatanov, D., and Gosselin, C., 2003. "Singularity analysis of 3-dof planar parallel mechanisms via screw theory". ASME Journal of Mechanical Design, 125(3), pp. 573-581.

[2] Gosselin, C., and Merlet, J., 1994. "On the direct kinematics of planar parallel manipulators: special architectures and number of solutions". Mechanism and Machine Theory, 29(8), pp. 1083-1097.

[3] Merlet, J., 2006. Parallel Robots. Springer.

[4] Wenger, P., and Chablat, D., 1998. "Workspace and assembly-modes in fully-parallel manipulators: a descriptive study". In Advances in Robot Kinematics and Computational Geometry, Kluwer Academic Publishers, pp. 117126.

[5] Borrel, P., 1986. "A study of manipulator inverse kinematic solutions with application to trajectory planning and workspace determination”. In Proc. IEEE Int. Conf on Rob. And Aut., pp. 1180-1185.

[6] Wenger, P., and Chablat, D., 1997. "Definition sets for the direct kinematics of parallel manipulators". In 8th International Conference in Advanced Robotics, pp. 859-864.

[7] Innocenti, C., and Parenti-Castelli, V., 1992. "Singularityfree evolution from one configuration to another in serial and fully-parallel manipulators". In Proc. ASME Design Technical Conferences, DE-Vol. 45, Spatial mechanisms and mechanical systems, Vol. 45, pp. 553-560.

[8] Hernandez, A., Altuzarra, O., Petuya, V., and Macho, E., 2009. "Defining conditions for non singular transitions between assembly modes". IEEE Transactions on Robotics, 25, p. 14381447.

[9] Hunt, K. H., and Primrose, E. J. F., 1993. "Assembly configurations of some in-parallel-actuated manipulators". Mechanism and Machine Theory, 28(1), p. 3142.

[10] Innocenti, C., and Parenti-Castelli, V., 1998. "Singularityfree evolution from one configuration to another in serial and fully-parallel manipulators". Journal of Mechanical Design, 120(1), p. 7379.

[11] Mcaree, P., and Daniel, R., 1999. “An explanation of neverspecial assembly changing motions for 3-3 parallel manipulators". The International Journal of Robotics Research, 18(6), pp. 556-574.

[12] Kong, X., and Gosselin, C., 2005. "Type synthesis of 5dof parallel manipulators based on screw theory". J. Field Robotics, 22(10), p. 535547.
[13] E. Macho, O. Altuzarra, C. P., and Hernandez, A., 2007. "Singularity free change of assembly mode in parallel manipulators. application to the 3-rpr planar platform". In 12th IFToMM World Congr., Besancon, France.

[14] Husty, M., 2009. "Non-singular assembly mode change in 3-rpr-parallel manipulators". In Computational Kinematics: Proceedings of the 5th International Workshop on Computational Kinematics, S. Verlag, ed., pp. 51-60.

[15] Zein, M., Wenger, P., and Chablat, D., 2007. "Singular curves in the joint space and cusp points of 3-rpr parallel manipulators". Robotica, 25(6), pp. 717-724.

[16] Wenger, P., 2004. "Uniqueness domains and regions of feasible paths for cuspidal manipulators". IEEE Transactions on Robotics, 20(4), pp. 745-750.

[17] Chablat, D., and Wenger, P., 2001. "Separation des solutions aux modeles geometriques direct et inverse pour les manipulateurs pleinement paralleles". Mechanism and Machine Theory, 36(6), pp. 763-783.

[18] Bamberger, H., Wolf, A., and Shoham, M., 2008. "Assembly mode changing in parallel mechanisms". IEEE Transactions on Robotics, 24(4), p. 765772.

[19] Caro, S., Moroz, G., Gayral, T., Chablat, D., and Chen, C., 2010. "Singularity analysis of a six-dof parallel manipulator using grassmann-cayley algebra and groebner bases". In Symposium on Brain, Body and Machine, Montreal, QC., Canada.

[20] Chen, C., Jackson, D., and Heyne, W., 2010. "A new 6-dof 3-legged parallel mechanism for forcefeedback interface". In Proceeding of the 2010 IEEE/ASME International Conference on Mechatronic and Embedded Systems and Applications, July, Qingdao.

[21] Bonev, I. A., and Ryu, J., 1999. "Orientation workspace analysis of 6-dof parallel manipulators". In ASME Design Engineering Technical Conferences.

[22] Lazard, D., and Rouillier, F., 2007. "Solving parametric polynomial systems”. J. Symb. Comput., 42(6), p. 636667.

[23] Moroz, G., Chablat, D., Wenger, P., and Rouiller, F., 2010. "Cusp points in the parameter space of rpr-2prr parallel manipulator". In 3-rd European Conference on Mechanism Science, Cluj-Napoca, Romania, Springer, pp. 29-37.

[24] Moroz, G., Rouiller, F., Chablat, D., and Wenger, P., 2010. "On the determination of cusp points of 3-rpr parallel manipulators". Mechanism and Machine Theory, 45(11), pp. $1555-1567$. 


\section{Appendix A: Singularities of the 3-PPPS-manipulator in its Joint Space}

The following equation corresponds to the singularities of the 3-PPPS-manipulator expressed in its joint space and is illustrated with Fig. 3.

$$
\begin{array}{r}
256-7600 Z_{2}^{6} Y_{1}^{4} X_{3}^{6}-7600 Z_{2}^{4} Y_{1}^{6} X_{3}^{6}-944 Z_{2}^{2} Y_{1}^{8} X_{3}^{6} \\
-944 Z_{2}^{6} Y_{1}^{2} X_{3}^{8} \\
+12048 Z_{2}^{4} Y_{1}^{4} X_{3}^{8}-944 Z_{2}^{2} Y_{1}^{6} X_{3}^{8}-1488 Z_{2}^{4} Y_{1}^{2} X_{3}^{10} \\
-1488 Z_{2}^{2} Y_{1}^{4} X_{3}^{10} \\
-592 Z_{2}^{2} Y_{1}^{2} X_{3}^{12}+18336 Z_{2}^{10} Y_{1}^{2} X_{3}^{2}-23712 Z_{2}^{8} Y_{1}^{4} X_{3}^{2} \\
+54112 Z_{2}^{6} Y_{1}^{6} X_{3}^{2} \\
-23712 Z_{2}^{4} Y_{1}^{8} X_{3}^{2}+18336 Z_{2}^{2} Y_{1}^{10} X_{3}^{2}-23712 Z_{2}^{8} Y_{1}^{2} X_{3}^{4} \\
-16608 Z_{2}^{6} Y_{1}^{4} X_{3}^{4} \\
-16608 Z_{2}^{4} Y_{1}^{6} X_{3}^{4}-23712 Z_{2}^{2} Y_{1}^{8} X_{3}^{4}+54112 Z_{2}^{6} Y_{1}^{2} X_{3}^{6} \\
-16608 Z_{2}^{4} Y_{1}^{4} X_{3}^{6}+54112 Z_{2}^{2} Y_{1}^{6} X_{3}^{6}-23712 Z_{2}^{4} Y_{1}^{2} X_{3}^{8} \\
-23712 Z_{2}^{2} Y_{1}^{4} X_{3}^{8}+18336 Z_{2}^{2} Y_{1}^{2} X_{3}^{10} \\
-11544 Z_{2}^{8} Y_{1}^{2} X_{3}^{2}-33420 Z_{2}^{6} Y_{1}^{4} X_{3}^{2}-33420 Z_{2}^{4} Y_{1}^{6} X_{3}^{2} \\
-11544 Z_{2}^{2} Y_{1}^{8} X_{3}^{2} \\
-33420 Z_{2}^{6} Y_{1}^{2} X_{3}^{4}+154584 Z_{2}^{4} Y_{1}^{4} X_{3}^{4}-33420 Z_{2}^{2} Y_{1}^{6} X_{3}^{4} \\
-33420 Z_{2}^{4} Y_{1}^{2} X_{3}^{6} \\
-33420 Z_{2}^{2} Y_{1}^{4} X_{3}^{6}-11544 Z_{2}^{2} Y_{1}^{2} X_{3}^{8}+83704 Z_{2}^{6} Y_{1}^{2} X_{3}^{2} \\
-29568 Z_{2}^{4} Y_{1}^{4} X_{3}^{2} \\
+83704 Z_{2}^{2} Y_{1}^{6} X_{3}^{2}-29568 Z_{2}^{4} Y_{1}^{2} X_{3}^{4}-29568 Z_{2}^{2} Y_{1}^{4} X_{3}^{4} \\
+83704 Z_{2}^{2} Y_{1}^{2} X_{3}^{6} \\
-42396 Z_{2}^{2} Y_{1}^{4} X_{3}^{2}-42396 Z_{2}^{2} Y_{1}^{2} X_{3}^{4}-592 Z_{2}^{12} Y_{1}^{2} X_{3}^{2} \\
-1488 Z_{2}^{10} Y_{1}^{4} X_{3}^{2} \\
-944 Z_{2}^{8} Y_{1}^{6} X_{3}^{2}-944 Z_{2}^{6} Y_{1}^{8} X_{3}^{2}-1488 Z_{2}^{4} Y_{1}^{10} X_{3}^{2} \\
-592 Z_{2}^{2} Y_{1}^{12} X_{3}^{2} \\
+1400 Y_{1}^{10}-1919 Y_{1}^{8}+16 Z_{2}^{16}+16 Y_{1}^{16}-2800 X_{3}^{6}-1919 X_{3}^{8} \\
+1488 Z_{2}^{10} Y_{1}^{2} X_{3}^{4}+12048 Z_{2}^{8} Y_{1}^{4} X_{3}^{4}-7600 Z_{2}^{6} Y_{1}^{6} X_{3}^{4} \\
+12048 Z_{2}^{4} Y_{1}^{8} X_{3}^{4} \\
+1488 Z_{2}^{2} Y_{1}^{10} X_{3}^{4}-944 Z_{2}^{8} Y_{1}^{2} X_{3}^{6}+224 Z_{2}^{14}-2800 Y_{1}^{6} \\
+24 Y_{1}^{14}+1048 Y_{1}^{12} \\
-10
\end{array}
$$




\section{Appendix B: Cuspidal configurations of the 3-PPPS- manipulator}

Equations (7)-(10) characterize the cuspidal configurations of the 3-PPPS-manipulator in its joint space, namely, as a function of variables $X_{3}, Y_{1}$ and $Z_{2}$. The cusp points are depicted in Fig. 5.

$$
\begin{array}{r}
24 Z 2^{4} Y 1^{2}-704+24 Z 2^{2} Y 1^{4}+24 Z 2^{4} X 3^{2}- \\
144 Z 2^{2} Y 1^{2} X 3^{2}+ \\
24 Y 1^{4} X 3^{2}+24 Z 2^{2} X 3^{4}+24 Y 1^{2} X 3^{4}- \\
215 Z 2^{4}-82 Z 2^{2} Y 1^{2}- \\
215 Y 1^{4}-82 Z 2^{2} X 3^{2}-82 Y 1^{2} X 3^{2} \\
-215 X 3^{4}+584 Z 2^{2}+584 Y 1^{2}+584 X 3^{2}=0
\end{array}
$$

$$
\begin{array}{r}
8 Z 2^{6}-32+8 Y 1^{6}-24 Z 2^{2} Y 1^{2} X 3^{2}+8 X 3^{6}- \\
37 Z 2^{4}+10 Z 2^{2} Y 1^{2}-37 Y 1^{4}+ \\
10 Z 2^{2} X 3^{2}+10 Y 1^{2} X 3^{2}-37 X 3^{4}+ \\
40 Z 2^{2}+40 Y 1^{2}+40 X 3^{2}=0
\end{array}
$$

$$
\begin{array}{r}
138768+36888 Z 2^{4}-125880 Z 2^{2}+3096 Y 1^{4}- \\
87480 Y 1^{2}+272864 X 3^{4}-326456 X 3^{2}+15648 Y 1^{6}- \\
109115 X 3^{6}+17712 X 3^{8}-1152 Y 1^{8}+384 X 3^{10}- \\
13152 Z 2^{2} Y 1^{4}-70619 Z 2^{4} X 3^{2}- \\
51227 Y 1^{4} X 3^{2}-119722 Z 2^{2} X 3^{4}- \\
87178 Y 1^{2} X 3^{4}+33264 Z 2^{2} Y 1^{2}+ \\
236984 Z 2^{2} X 3^{2}+183992 Y 1^{2} X 3^{2}+ \\
21720 Z 2^{2} X 3^{6}-8976 Y 1^{6} X 3^{2}+ \\
29832 Y 1^{4} X 3^{4}+12744 Y 1^{2} X 3^{6}+ \\
32232 Z 2^{4} X 3^{4}-2112 Y 1^{2} X 3^{8}- \\
3072 Z 2^{4} X 3^{6}-2688 Z 2^{2} X 3^{8}+ \\
576 Y 1^{8} X 3^{2}+576 Y 1^{6} X 3^{4}- \\
2496 Y 1^{4} X 3^{6}-26278 Z 2^{2} Y 1^{2} X 3^{2}- \\
24360 Z 2^{2} Y 1^{2} X 3^{4}+18096 Z 2^{2} Y 1^{4} X 3^{2}- \\
5760 Z 2^{2} Y 1^{4} X 3^{4}+14592 Z 2^{2} Y 1^{2} X 3^{6}=0
\end{array}
$$

$$
\begin{array}{r}
-151136-42611 Z 2^{4}+133592 Z 2^{2}-11699 Y 1^{4}+ \\
97496 Y 1^{2}-123827 X 3^{4}+232664 X 3^{2}-12984 Y 1^{6}+ \\
28296 X 3^{6}+576 X 3^{8}+576 Y 1^{8}+9768 Z 2^{2} Y 1^{4}+ \\
41280 Z 2^{4} X 3^{2}+38064 Y 1^{4} X 3^{2}+25512 Z 2^{2} X 3^{4}+ \\
12528 Y 1^{2} X 3^{4}-23578 Z 2^{2} Y 1^{2}-135706 Z 2^{2} X 3^{2}- \\
104794 Y 1^{2} X 3^{2}-4032 Z 2^{2} X 3^{6}+1152 Y 1^{6} X 3^{2}-- \\
3456 Y 1^{4} X 3^{4} 3456 Y 1^{2} X 3^{6}-4608 Z 2^{4} X 3^{4}+576 Z 2^{2} Y 1^{6}- \\
4440 Z 2^{2} Y 1^{2} X 3^{2}+22464 Z 2^{2} Y 1^{2} X 3^{4}- \\
9792 Z 2^{2} Y 1^{4} X 3^{2}=0
\end{array}
$$

